\title{
Local helioseismology and the active Sun
}

\author{
H. Schunker * \\ Max-Planck-Institut für Sonnensystemforschung, Max-Planck-Strasse 2, Katlenburg-Lindau, 37191, Germany
}

Received 30 May 2005, accepted 11 Nov 2005

Published online later

Key words Sun: helioseismology; oscillations; sunspots

The goal of local helioseismology is to elicit three-dimensional information about the sub-surface (or far-side) structure and dynamics of the Sun from observations of the helioseismic wave field at the surface. The physical quantities of interest include flows, sound-speed deviations and magnetic fields. However, strong surface magnetic fields induce large perturbations to the waves making inversions difficult to interpret. The purpose of this paper is to outline the methods of analysis used in local helioseismology, review discoveries associated with the magnetic Sun made using local helioseismology from the past three years, and highlight the efforts towards imaging the interior in the presence of strong magnetic fields.

(c) 2006 WILEY-VCH Verlag GmbH \& Co. KGaA, Weinheim

\section{Introduction}

Helio- and astero-seismology provide the means to "see" beneath the surface of the Sun or star, and to gain an understanding of the physics of stellar interiors. The analysis of the frequencies of the global modes of oscillation allows us to study the internal stellar structure only as a function of radius and unsigned latitude. With high spatial resolution observations of the velocity signal on the Sun's surface, local helioseismology is able to image the three dimensional subsurface structure of the Sun and image magnetic activity on the far-side. Local helioseismology aims to interpret the full wave field observed at the solar surface, not only the frequencies of the normal modes. We restrict our attention, here, to local helioseismology.

The Doppler signal, which is the line-of-sight velocity of the solar surface, is the fundamental observable. Full-disk high-quality data is obtained from instruments including the Michelson Doppler Image (MDI) onboard the Solar and Heliospheric Observatory (SOHO) (Scherrer et al. 1995), the Global Oscillation Network Group (GONG) (Harvey et al. 1996), and the Helioseismic and Magnetic Imager (HMI) onboard the recently launched Solar Dynamics Observatory (SDO). MDI also runs higher-resolution campaigns on smaller fields-of-view. The Magneto-Optical filter at Two Heights (Finsterle et al. 2004) observes simultaneously at two heights in the atmosphere allowing an analysis of the vertical propagation of waves.

Various local helioseismic techniques exist to analyse the oscillations. The main ones are time-distance analysis (Duvall et al. 1993), ring diagram analysis (Hill 1988) and acoustic imaging/holography (Chang et al. 1997; Lindsey \& B 1997). Ring-diagram analysis is most closely related to global

\footnotetext{
^ Corresponding author: e-mail: schunker@mps.mpg.de
}

mode helioseismology. By analysing frequency shifts over small regions of the solar surface, the direction and amplitude of subsurface flows can be determined. Time-distance analysis computes the travel time of a wave packet travelling between two points on the solar surface. Helioseismic holography (acoustic imaging) reconstructs the subsurface wave fields by propagating waves either forward or backward in time. Other methods include Fourier-Hankel analysis (Braun et al. 1987), designed specifically to analyse waves surrounding a sunspot. Woodard (2009) used direct modelling to interpret the wave correlations in wavevectorfrequency space.

The forward problem is then to compute the expected seismic observations from a particular model of the Sun. The inverse problem is to infer the internal properties of the Sun from the seismic observables. Local helioseismology is the only way to elicit the three-dimensional structure of the solar interior, including structures and flows on a range of scales.

In this paper we focus on the local helioseismology of sunspots, active regions and large scale flows varying with the solar cycle. There are particular problems associated with doing helioseismology in the immediate vicinity of strong surface magnetic fields. The forward problem used to infer the subsurface properties has previously been based exclusively on solving hydrodynamic equations in the absence of a magnetic field. Additionally, the actual inversion method assumes that any perturbation to the waves is small, however it has been demonstrated that strong surface magnetic fields cause large perturbations to the waves. Knowing the subsurface structure of sunspots and active regions Bratillf fonstrain sunspot models and hopefully determine their deeper structure. The immediate need now is to quantitatively model wave propagation through magnetic fields and, subsequently, account for the effects when inverting for the subsurface structure. 
An important question in solar physics is to understand the mechanics of the solar dynamo. Local helioseismology has identified large scale flow variations that appear to be intimately connected to the surface activity. Understanding such connections will help to decipher the larger scale dynamics of the magnetic Sun. Other goals of local helioseismology are to measure the meridional flow and changes at the base of the convection zone, which would help constrain models of the dynamo.

This short review will cover the basic techniques used in local helioseismology in Section 2. How the interior structure is subsequently inferred is described in Section 3 . Section 4 will then cover some of the results, over the past three years, from local helioseismic analysis tied to solar activity. A concentrated effort towards modelling waves propagating through magnetic fields to understand the observations will be reviewed in Section 5 Recent reviews of local helioseismology include Birch (2008); Gizon \& Thompson (2007); Komm et al. (2006). For more extensive reviews of local helioseismology see, for example, Gizon et al. (2010).

\section{Analysis techniques}

\subsection{Ring-diagram analysis}

Typically ring-diagram analysis uses many small regions covering a significant fraction of the visible solar surface. Beneath each patch, two independent quantities, e.g. sound speed and density, may be inferred as a function of depth from which other quantities can be calculated. By combining the information in each patch, a three-dimensional picture is achieved. Typically this method is used to probe the top $30 \mathrm{Mm}$ of the convection zone.

For each patch a three-dimensional power spectrum is computed. A cut of the power spectrum at constant frequency results in an image of rings (Hill 1988).

Variations in the power spectra indicate particular subsurface properties. The presence of a flow shifts introduces a Doppler frequency shift and affects the shape of the rings. The degree to which the different modes are affected gives some indication of the depth and strength of the flow. Cuts at constant wavenumber can also be considered, where the power spectrum is a function of azimuthal angle and frequency. The measurement technique used in ring-diagram analysis and their interpretation are discussed in (e.g. Basu et al. 1999; Haber et al. 2000).

\subsection{Time-distance}

Time-distance helioseismology (Duvall et al.1993) involves measuring the (phase) travel-time between two points on the solar surface from the cross-covariance function of the Doppler signal at the two points. The cross-covariance is sensitive to the wave speed in the solar interior. For example, if there is a horizontal flow in the direction from point 1 to point 2 then this will result in a reduced travel time, and a longer travel time if the flow is in the opposite direction. One way to extract the travel times from the crosscovariance is to fit Gabor wavelets to the cross-covariance (Duvall et al. 1997) as a function of time-lag. The crosscovariance can also be fit using a one parameter fit Gizon \& Birch (2002, 2004). Often, the cross-covariance is computed between a central point and a surrounding annulus, and averaged over the annulus. This technique is used to study the divergence of flows. The direction of the flows is gained by dividing the annulus into quadrants (e.g. Duvall et al. 1996).

Filtering the observations is a common practice. In ridge filtering only modes of a particular radial order are retained, and in phase-speed filtering waves within a certain range of wave speed (or depth penetration) are retained. It has recently come to light that the filtering process has a strong effect on the travel-time measurements and must be included in any interpretation of the observations (Braun \& Birch 2008; Gizon et al. 2009; Moradi et al. 2009b).

\subsection{Acoustic imaging/holography}

Acoustic imaging (Chang et al. 1997) and helioseismic holography (Lindsey \& Braun 1997) take the surface Doppler observations and reconstruct the subsurface wave field from ingoing and outgoing waves with respect to a pupil. The ingoing waves are propagated forward in time and the outgoing waves are propagated backward in time using Green's functions and a knowledge of the solar interior. Comparisons between the forward and backward propagating waves are used to derive phase shifts and travel time variations. Far-side helioseismology (Lindsey \& Braun 2000) was developed using this method, although it is also possible with time-distance techniques (Zhao 2007).

\subsection{Fourier-Hankel}

Specifically designed with the intention to study waves surrounding sunspots, Fourier-Hankel analysis (Braun et al.1987) decomposes the observed oscillations into in- and out-going waves in a cylindrical coordinate system centred on a sunspot. The observed waves are observed in an annulus surrounding the sunspot. From this the in- and out-going waves can be compared, in particular, their amplitude and phases (Braun 1995; Braun et al. 1988).

\section{Inversions}

The inverse problem in helioseismology involves extracting quantitative physical information regarding Solar subsurface inhomogeneities; such as sound-speed, density, mass flows, and magnetic field. This requires accurate and realistic forward modelling of the solar oscillations, given a prescribed background model. Model $\mathrm{S}$ (Christensen-Dalsgaard et al. 1996) is often used for this purpose in helioseismology. Furthermore, robust and reliable helioseismic travel-time measurements must be combined, or averaged, in some sensible way in order to best 
exploit the physical quantities of interest. It is also very important that the noise properties of the observations be well understood and accounted for (Gizon \& Birch 2004).

Heretofore, the effect of the inhomogeneities on the oscillations has been assumed to be weak. This ultimately yields a linear integral relationship relating observations, 3D sensitivity kernel functions, and the subsurface perturbations. As yet, no non-linear inversions have been attempted.

There exist two primary procedures for computing inversions for time-distance and ring-diagram analysis; The Regularised Least Squares (RLS) method (e.g. Kosovichev 1996) and the Optimally Localised Averaging (OLA) method (e.g. Jackiewicz et al. 2007; Pijpers \& Thompson 1992

Accurate inversions depend heavily on the accuracy of the kernel functions, which must reflect all the aspects of the measurement procedure, including filtering. The ray approximation can be used to calculate the kernels Kosovichev 1996), but it is only useful if the background properties do not vary on scales smaller than, or comparable to, the wavelength. The first-order Born approximation offers a more reliable way to compute kernels, since it accounts for finite wavelength effects (Birch \& Felder 2004; Gizon \& Birch 2002; Hung et al. 2001). Recently, Birch et al. (2007) have computed sensitivity kernels for ring-diagram analysis and Birch \& Gizon (2007) have computed Born kernels for time-distance helioseismology.

Jackiewicz et al. (2008) have recently obtained maps of supergranular vector flows in the near-surface layers using an OLA inversion of travel times

\section{Recent observational results}

\subsection{Meridional flow}

Meridional flow is the flow from the Equator towards the poles near the surface, with a return flow at some depth. The meridional flow is important for magnetic flux transport in solar dynamo models. An estimate of the meridional flow at the base of the convection zone predicts an amplitude of a few $\mathrm{m} \mathrm{s}^{-1}$ whereas the sound speed at this depth is a few thousand $\mathrm{m} \mathrm{s}^{-1}$, making it difficult to detect as discussed by Braun \& Birch (2008).

Recently Gizon \& Rempel (2008) and González Hernández et al. (2008) have measured the mean amplitude of the flow using time-distance and ring-diagram techniques respectively and get consistent values of $10-15$ $\mathrm{m} \mathrm{s}-1$ for the mean meridional flow near the surface.

\subsection{Solar cycle variations of meridional flow}

The meridional flow varies with the solar cycle. The residual from the mean flows at each latitude is a $\pm 5 \mathrm{~m} \mathrm{~s}^{-} 1$ converging flow towards the active region belt that increases in strength with increasing activity (Gizon \& Rempel 2008; González Hernández et al. 2008).
It is known that active regions are surrounded at the surface by a general inflow and when averaged longitudinally these flows introduce a perturbation to the meridional circulation. Spruit (2003) suggested that the inflow is due to the increased radiative loss of the active region compared to the surrounding Sun, and this can explain the solar cycle variations of the meridional flow. In agreement with this, it has been shown by Gizon \& Rempel (2008) that a model including increased radiative loss in the active regions can reproduce the inflows and explain the observed surface solarcycle variations.

By masking out active regions and their surrounding flows, Gizon (2004) finds that the influence on longitudinal averages of active region flows is significantly reduced. This implies that the flows surrounding active regions are the major contributors to the time varying component of the meridional flow. Recently, González Hernández et al. (2010) have shown that in this extended solar minimum the residual meridional flow developed before the onset of visible magnetic activity, suggesting that there is still a component independent of the presence of active regions.

\subsection{Torsional oscillations}

Torsional oscillations are bands of slightly faster rotation coinciding with the activity belt which migrate towards the equator over a solar cycle. The flow band associated with the new solar cycle has been found to be moving more slowly than in the lead up to the previous cycle (Howe et al. 2009), possibly providing a clue as to why the onset of Solar Cycle 24 is later than expected.

\subsection{Sunspots}

There is considerable observational evidence that sunspots have a large effect on waves. It is well documented that sunspots absorb up to $50 \%$ of the energy and shift the phase of incoming waves (Braun et al. 1988, 1992). Various dependencies on frequency and radial order were also found. These results were originally discovered using Fourier-Hankel methods.

In the past few years, more evidence has emerged that the wave signal is dominated by surface effects (e.g. Braun \& Birch 2008; Cally 2009; Gizon et al. 2009). Full waveform forward modelling has also revealed that a shallow sunspot model is sufficient to quantitatively explain the observed change in amplitude and phase of the waves in caused by a sunspot (Cameron et al. 2010). Despite the accumulating evidence that sunspots are large perturbations to the waves, wave-speed inversions below sunspots have traditionally been done under simplifying assumptions (weak and non-magnetic perturbations). Such results should be treated with caution due to the inherent problems of attempting a linear inversion involving non-linear perturbations to the waves.

Additionally, the inversions do not account for source suppression or details of the measurement procedure (e.g. 
Braun \& Birch 2008, show that the filtering is crucial). Figure 19 from Moradi et al. (2009a) directly compares the results of various sunspot inversions. All of the methods, except the inversion for time-distance analysis, appear to be consistent with an increasing wave-speed in the top $2 \mathrm{Mm}$ and small perturbations below this. However, this does not mean either result is correct and the cause(s) of the discongruity needs to be fully understood before a reliable inversion can be made.

For a more comprehensive review of sunspot seismology see e.g. Gizon et al. (2009), and for sunspot models in local helioseismology Moradi et al. (2009a).

\subsection{Acoustic power and magnetic activity}

It has been known for some time that acoustic power is altered by the presence of magnetic field. Suppression of acoustic power is evident in strong magnetic fields consistent with reduced convective sources. This suppression affects the computed cross-covariances and thus the travel times.

It has also been observed that higher frequency $(5-6.5 \mathrm{mHz})$ wave power is enhanced in regions surrounding active regions and sunspots (Hindman \& Brown 1998), called acoustic halos. Recently, Schunker \& Braun (2010) found that the halos tend to be stronger in regions of intermediate strength horizontal magnetic field. From such a basic relationship between the acoustic power and magnetic field vector, they are able to reproduce the acoustic power maps from the vector magnetic field to a high degree. They also found that the high frequency ridges of the power spectrum are shifted to higher wavenumber. Several mechanisms have been suggested for generating the halo although none reproduce all of the observed characteristics of the halo. The mechanisms include mode conversion (Khomenko \& Collados 2009), mode scattering (Hanasoge 2009), trapping under magnetic field canopies (Kuridze et al. 2008), and a change in convective scales (Jacoutot et al. 2008).

Chou et al. (2009) analysed the spatial distribution of acoustic emission, absorption and suppression characteristics of two active regions and found that they all correlate spatially with the magnetic field, including the plage regions.

Zharkovetal. (2009) observe enhanced power in sunspot umbra at large line-of-sight angles, i.e. perpendicular to the field. They model the propagation of waves through localised magnetic field and conclude that it is likely they are observing slow modes.

\section{Recent theoretical developments}

To understand the seismic signal observed on the surface of the Sun it is essential to model the propagation of waves through a magnetic field. There are three ways that this has been done: $i$ ) with simplified models aimed at understanding the physical mechanisms; $i i)$ with realistic numerical simulations of magnetoconvection where waves are naturally excited by convection; and iii) with numerical simulations of linear wave propagation through a prescribed static background.

The models of $i$ ) were instrumental for discerning the relevant physics. Spruit \& Bogdan (1992) proposed that the absorption could be caused by mode conversion, and subsequent studies explored the physics (e.g. Cally \& Bogdan 1993, 1997). In fact, Crouch et al. (2005) demonstrated that mode conversion could explain both the observed absorption and phase shifts and match the quantitative measurements quite well (Cally et al.|2003). These models suggested that the mode conversion occurs in the top $1 \mathrm{Mm}$ below the surface, where the Alfvén speed is comparable to the sound speed. More recently, Cally (2009) has shown that in umbrae thermal effects are likely to dominate the waves, whereas in the penumbra magnetic effect dominate. While these important results elucidate the mechanisms, now we require more realistic simulations where we can also test the analysis techniques and quantitatively measure the effects.

Approach ii) is fully physical, but very computer intensive. These kinds of simulations allow a study of the behaviour of granulation, and thus changes in the properties of the waves, in the presence of magnetic fields. The most recent simulations including a sunspot can be seen in Rempel et al. (2009), although limited helioseismic analysis has been published (Birch et al. 2009).

The third approach is less computer intensive and allows for parametric studies of magnetic structures. A disadvantage is that these simulations require a stable background model. Several groups have developed numerical codes designed to model oscillations in the vicinity of sunspots using linearised MHD equations:

- The IAC MHD code (Khomenko et al. 2008) has been used for a variety of studies. Some of the relevant results include that seismic waves below a sunspot travel faster than in the quiet-Sun and that high-frequency fast waves refracted by the magnetic field just above the surface are possibly the cause of acoustic halos Khomenko \& Collados 2009).

- The SLiM code (Cameron et al. 2007) together with a carefully constructed stable background (Schunker et al. 2010a) and sunspot model is able to reproduce observational results (Cameron et al. 2010). The sunspot model itself was also somewhat constrained by the comparison (Cameron et al. 2008). The code has also been used to study the separate effects of the magnetic field and thermal effects of a sunspot.

- The SPARC code (Hanasoge et al. 2006) has been used to study waves surrounding magnetic flux tubes, in particular the acoustic halo (Hanasoge 2009).

- The SAC code (Shelyag et al. 2008, 2009) was used to analyse the propagation and dispersion of waves in a non-uniform magnetic field. They also found that the largest contribution to travel time perturbations was due 
to the thermal structure in weak to intermediate magnetic field strengths, but that mode conversion in strong fields is important.

All of these codes give qualitatively similar results.

A careful analysis of the effects of stabilising a solar model against convection has been done using the SLiM code (Schunker et al. 2010b). The stabilisation procedure changes the properties of the model, and in turn alters the eigenmodes. Schunker et al. (2010b) attempt to correct for changes to the eigenmodes by modifying the background model. Since the models are stable, a model of the convective sources needs to be implemented as well as a model of the damping of the waves. All of these parameters need to work in concert to produce seismically solar-like simulations to provide realistic interpretations of the helioseismic observations.

\section{Discussion and future studies}

Further confirmation of the surface effects of strong magnetic fields have been found in observations, casting further doubt on over-simplified inversions for wave-speed and flows below magnetic active regions and sunspots. The effects of filtering, source suppression and mode conversion of the waves in strong surface magnetic fields have yet to be fully quantified for each of the analysis techniques. In turn, a concerted effort is underway to quantitatively simulate the seismic signature of the Sun in the presence of magnetic fields to explore these effects. The simulations are still being fully analysed and in the near future a quantitative measure of the effects may be used to infer the correct subsurface properties of a sunspot.

Future efforts in local helioseismology will focus on deeper inversions, particularly of the base of the convection zone, and steps towards this have been taken using timedistance techniques (Zhao et al. 2009).

A challenging goal is to detect the emergence of magnetic flux before it is observed at the surface. Some progress has been made towards this (e.g. Hartlep et al. 2010; Komm et 2009, 2008), but strong definitive signatures have not been observed. Future studies of the effects of magnetic flux emergence will require systematic helioseismic analysis of many emerging active regions and realistic simulations.

The HMI instrument onboard the recently launched SDO very soon provide increased resolution observations of the seismic Sun and provide accompanying vector magnetograms on a regular basis. Thanks to the improved spatial resolution we anticipate being able to do local helioseismology closer to the limb (and poles).

Acknowledgements. HS was supported by the European Helioand Asteroseismology Network (HELAS), a major international collaboration funded by the European Commission's Sixth Framework Programme.

\section{References}

Basu, S., Antia, H. M., \& Tripathy, S. C. 1999, ApJ , 512, 458

Birch, A., Braun, D. C., \& Rempel, M. 2009, in AAS/Solar Physics Division Meeting, Vol. 40, AAS/Solar Physics Division Meeting

Birch, A. C. 2008, Journal of Physics Conference Series, 118, 12009

Birch, A. C. \& Felder, G. 2004, ApJ , 616, 1261

Birch, A. C. \& Gizon, L. 2007, Astronomische Nachrichten, 328, 228

Birch, A. C., Gizon, L., Hindman, B. W., \& Haber, D. A. 2007, ApJ , 662, 730

Braun, D. C. 1995 , ApJ , 451, 859

Braun, D. C. \& Birch, A. C. 2008, Solar Phys., 251, 267

Braun, D. C., Duvall, Jr., T. L., \& Labonte, B. J. 1987, ApJ , 319, L27

Braun, D. C., Duvall, Jr., T. L., \& Labonte, B. J. 1988, ApJ , 335, 1015

Braun, D. C., Lindsey, C., Fan, Y., \& Jefferies, S. M. 1992, ApJ , 392, 739

Cally, P. S. 2009, MNRAS , 395, 1309

Cally, P. S. \& Bogdan, T. J. 1993, ApJ , 402, 721

Cally, P. S. \& Bogdan, T. J. 1997, ApJ , 486, L67

Cally, P. S., Crouch, A. D., \& Braun, D. C. 2003, MNRAS , 346, 381

Cameron, R., Gizon, L., \& Daiffallah, K. 2007, Astronomische Nachrichten, 328, 313

Cameron, R., Gizon, L., \& Duvall, Jr., T. L. 2008, Solar Phys., 251, 291

Cameron, R., Gizon, L., Schunker, H., \& Pietarila, A. 2010, ArXiv e-prints: 1003.0528

Chang, H., Chou, D., Labonte, B., \& The TON Team. 1997, Nature, 389, 825

Chou, D., Yang, M., Zhao, H., Liang, Z., \& Sun, M. 2009, ApJ , 706, 909

Christensen-Dalsgaard, J., Dappen, W., Ajukov, S. V., et al. 1996, Science, 272, 1286

Crouch, A. D., Cally, P. S., Charbonneau, P., Braun, D. C., \& Desjardins, M. 2005, MNRAS , 363, 1188

Duvall, Jr., T. L., Jefferies, S. M., Harvey, J. W., \& Pomerantz, M. A. 1993, Nature, 362, 430

vall, Jr., T. L., Kosovichev, A. G., Scherrer, P. H., et al. 1997, Solar Phys., 170, 63

Duvall, Jr., T. L., Kosovichev, A. G., Scherrer, P. H., \& Milford, P. N. 1996, in Bulletin of the American Astronomical Society, Vol. 28, Bulletin of the American Astronomical Society, 898

Finsterle, W., Jefferies, S. M., Cacciani, A., Rapex, P., \& McIntosh, S. W. 2004, ApJ , 613, L185

Gizon, L. 2004, Solar Phys., 224, 217

Gizon, L. \& Birch, A. C. 2002, ApJ , 571, 966

Gizon, L. \& Birch, A. C. 2004, ApJ , 614, 472

Gizon, L., Birch, A. C., \& Spruit, H. C. 2010, ArXiv eprints: 1001.0930

Gizon, L. \& Rempel, M. 2008, Solar Phys., 251, 241

Gizon, L., Schunker, H., Baldner, C. S., et al. 2009, Space Science Rev., 144, 249

Gizon, L. \& Thompson, M. J. 2007, Astronomische Nachrichten, 328,204

González Hernández, I., Howe, R., Komm, R., \& Hill, F. 2010, ApJ , 713, L16 
González Hernández, I., Kholikov, S., Hill, F., Howe, R., \& Komm, R. 2008, Solar Phys., 252, 235

Haber, D. A., Hindman, B. W., Toomre, J., et al. 2000, Solar Phys., 192,335

Hanasoge, S. M. 2009, Astron. Astrophys., 503, 595

Hanasoge, S. M., Larsen, R. M., Duvall, Jr., T. L., et al. 2006, ApJ , 648,1268

Hartlep, T., Kosovichev, A. G., Zhao, J., \& Mansour, N. N. 2010, ArXiv e-prints: 1003.4305

Harvey, J. W., Hill, F., Hubbard, R. P., et al. 1996, Science, 272, 1284

Hill, F. 1988, ApJ , 333, 996

Hindman, B. W. \& Brown, T. M. 1998, Astrophys. J., 504, 1029

Howe, R., Christensen-Dalsgaard, J., Hill, F., et al. 2009, ApJ , 701, L87

Hung, S., Dahlen, F. A., \& Nolet, G. 2001, Geophysical Journal International, 146, 289

Jackiewicz, J., Gizon, L., \& Birch, A. C. 2008, Solar Phys., 251, 381

Jackiewicz, J., Gizon, L., Birch, A. C., \& Thompson, M. J. 2007, Astronomische Nachrichten, 328, 234

Jacoutot, L., Kosovichev, A. G., Wray, A., \& Mansour, N. N. 2008, ApJ , 684, L51

Khomenko, E. \& Collados, M. 2009, Astron. Astrophys., 506, L5

Khomenko, E., Collados, M., \& Felipe, T. 2008, Solar Phys., 251, 589

Komm, R., Howe, R., \& Hill, F. 2006, Advances in Space Research, 38, 845

Komm, R., Howe, R., \& Hill, F. 2009, Solar Phys., 258, 13

Komm, R., Morita, S., Howe, R., \& Hill, F. 2008, ApJ , 672, 1254

Kosovichev, A. G. 1996, ApJ , 461, L55

Kuridze, D., Zaqarashvili, T. V., Shergelashvili, B. M., \& Poedts, S. 2008, Ann. Geophys., 26, 2983

Lindsey, C. \& Braun, D. C. 1997, ApJ , 485, 895

Lindsey, C. \& Braun, D. C. 2000, Science, 287, 1799

Moradi, H., Baldner, C., Birch, A. C., et al. 2009a, ArXiv eprints:0912.4982

Moradi, H., Hanasoge, S. M., \& Cally, P. S. 2009b, ApJ , 690, L72

Pijpers, F. P. \& Thompson, M. J. 1992, Astron. Astrophys., 262, L33

Rempel, M., Schüssler, M., Cameron, R. H., \& Knölker, M. 2009, Science, 325, 171

Scherrer, P. H., Bogart, R. S., Bush, R. I., et al. 1995, Solar Phys., 162,129

Schunker, H. \& Braun, D. C. 2010, Solar Phys., accepted

Schunker, H., Cameron, R., \& Gizon, L. 2010a, ArXiv eprints: 1002.1969

Schunker, H., Cameron, R., Gizon, L., \& Moradi, H. 2010b, Solar Phys., submitted

Shelyag, S., Fedun, V., \& Erdélyi, R. 2008, Astron. Astrophys., 486, 655

Shelyag, S., Zharkov, S., Fedun, V., Erdélyi, R., \& Thompson, M. J. 2009, Astron. Astrophys., 501, 735

Spruit, H. C. 2003, Solar Phys., 213, 1

Spruit, H. C. \& Bogdan, T. J. 1992, ApJ , 391, L109

Woodard, M. F. 2009, ApJ , 706, L62

Zhao, J. 2007, ApJ , 664, L139

Zhao, J., Hartlep, T., Kosovichev, A. G., \& Mansour, N. N. 2009, ApJ , 702, 1150

Zharkov, S., Shelyag, S., Erdélyi, R., \& Thompson, M. J. 2009, ArXiv e-prints 